\title{
Impact de la gestation et de non gestation sur I'hématocrite, hémoglobine et les teneurs martiales chez la chèvre a Lubumbashi en zone tropicale
}

\author{
Tshiasuma K. A ${ }^{1}$., Ngoie K2 Kaluendi C. E ${ }^{3}$ et Kasereka S. B ${ }^{*}$ \\ 1. Service de biologie générale, conservation de la nature et faune sauvage, faculté de Médecine Vétérinaire, Université \\ de Lubumbashi B.P. 1825 Lubumbashi, R.D.Congo \\ 2. Service de physiologie et physiopathologie faculté de Médecine Vétérinaire, Université de Lubumbashi B.P. 1825 \\ Lubumbashi, R.D.Congo \\ 3. Service de Biochimie normale et pathologique faculté de Médecine Vétérinaire, Université de Lubumbashi B.P. 1825 \\ Lubumbashi, R.D.Congo \\ 4. service d'Anatomie et Autopsie faculté de Médecine Vétérinaire, Université de Lubumbashi B.P. 1825 Lubumbashi, \\ R.D.Congo \\ Auteurs chargés de la correspondance : E-mail : bourg.2013@gmail.com
}

Original submitted in on $21^{\text {st }}$ September 2017. Published online at www.m.elewa.org on $28^{\text {th }}$ February 2018 https://dx.doi.org/10.4314/iab.v122i1.3

\section{RESUME}

Objectif : Évaluer l'impact de la gestation et de la non gestation sur l'hématocrite, hémoglobine et les teneurs martiales chez la chèvre à Lubumbashi en zone tropicale.

Méthodologie et résultats : 76 échantillons du sang total, du sang total hémolysé et de sérum de 38 chèvres exploitées à Lubumbashi dont 18 gestantes et 20 non gestantes ont été analysés. L'hématocrite dosé à partir de la micro centrifugeuse et le taux d'hémoglobine par la méthode de Sahli étaient respectivement de 35,33 \pm $6,98 \%$ et $6,23 \pm 1,64 \mathrm{~g} \%$ chez les gestantes et de $35,55 \pm 5,23 \%$ et $5,98 \pm 0,64 \mathrm{~g} \%$ chez les non gestantes. Les teneurs martiales du sang total hémolysé et du sérum dosé par test colorimétrique à partir du spectrophotomètre étaient respectivement de $80,47 \pm 32,39 \mu \mathrm{mol} / / \mathrm{l}$ et $11,76 \pm 13,9 \mu \mathrm{mol} / \mathrm{l}$ chez les gestantes et de $122,36 \pm 59,04 \mu \mathrm{mol} / /$ et $18,45 \pm 12,35 \mu \mathrm{mol} / / \mathrm{l}$ chez les non gestantes. Les teneurs martiales globulaires, écart entre le fer sanguin total et le fer sérique étaient de 68,71 $\pm 39,14 \mu \mathrm{mol} / \mathrm{I}$ chez les gestantes et de 103,50 $\pm 59,20 \mu \mathrm{mol} / \mathrm{l}$ chez les non gestantes. Statistiquement, la gestation n'a pas influencé l'hématocrite et le taux d'hémoglobine, mais la supériorité de toutes les teneurs martiales des non gestantes sur les gestantes étaient remarquable. Par rapport au fer sanguin total, dans le sérum et globules rouges, les teneurs martiales sont de l'ordre de $15 \%$ et $85 \%$.

Conclusion et application des résultats : En considérant le taux d'hémoglobine, ces chèvres sont anémiques, d'où la nécessité de les supplémenter en fer surtout pendant la gestation.

Mots clés : Impact, gestation, hématocrite, hémoglobine, teneur martiale, zone tropicale. 
Impact of gestation and non-gestation on packed cell, haemoglobin rate and iron rate of the goat of Lubumbashi in tropical area

\section{ABSTRACT}

Objective : To evaluate the impact of gestation and non-gestation on packed cell, hemoglobin rate and iron rate of the goat of Lubumbashi in tropical area.

Methodology and results : 76 samples of whole blood, haemolysed whole blood and serum of 38 goats, in Lubumbashi among which 18 pregnant and 20 non-pregnant were analyzed. Packed cell volume obtained after centrifugation and haemoglobin rate from Sahli method were respectively $35,33 \pm 6,98 \%$ and $6,23 \pm 1,64 \mathrm{~g} \%$ in the pregnant goats, $35,55 \pm 5,23 \%$ and $5,98 \pm 0,64 \mathrm{~g} \%$ in the non-pregnant goats. The iron rates of whole blood haemolysed and of serum were obtained from colorimetric test with spectrophotometer and were respectively $80,47 \pm 32,39 \mu \mathrm{mol} / \mathrm{l}$ and $11,76 \pm 13,9 \mu \mathrm{mol} / /$ for the pregnant, $122,36 \pm 59,04 \mu \mathrm{mol} / \mathrm{l}$ and 18 , $45 \pm 12,35 \mu \mathrm{mol} / /$ for the non-pregnant goats. The globular iron rate, the difference between total iron and serum iron were $68,71 \pm 39,14 \mu \mathrm{mol} / \mathrm{l}$ in the pregnant goats and 103, $50 \pm 59,20 \mu \mathrm{mol} / \mathrm{l}$ in the non-pregnant goats. Statistically, the pregnant did not influence the packed cell and the haemoglobin rate, but the all iron rate of non-pregnant goats were highest. According to the iron rate in the whole blood, in the serum and the red blood cells the iron rate was respectively $15 \%$ and $85 \%$.

Conclusion and application of results: These goats are thus anaemic, we recommend to the farmers to supplement the feed with more iron, especially for the pregnant

KEYWORDS: Pregnancy impact, Packed cell volume, Haemoglobin, Iron rate, Tropical area

\section{INTRODUCTION}

L'élevage des caprins, à cause de la rusticité de celui-ci et de la reproduction fréquemment gémellaire, révèle que le caprin est un investissement sûr à court, moyen et long terme (Lunumbi, 2008). Chez les caprins comme chez d'autres espèces, le fer bien qu'un oligo-élément, intervient dans plusieurs processus métaboliques et hématologiques (Barone, 2010; Marpeau, 2010; Suttle, 2010). Ainsi, les besoins en fer varient selon les états physiologiques de gestation, de non gestation et de lactation. (Ngoie et al, 2016). Dans le site d'élevage, la teneur en fer du sol et des pâturages varie énormément. Si l'élevage caprin présente des nombreux avantages, il existe néanmoins diverses contraintes. En effet, le prolongement de la saison sèche réduit les ressources alimentaires tout en favorisant les aliments toxiques; tout comme les intenses pluies qui s'accompagnent des expansions des verminoses, peuvent intensifier les carences en fer plus particulièrement chez les gestantes et entrainé ainsi des anémies (Makumyaviri et Kabamba, 2006). En élevage caprin, comme la supplémentation en fer n'est presque pas d'application, l'anémie ferriprive peut être très fréquente, surtout chez les gestantes et les chevreaux (Ndoutamia et Ganda, 2005). Ainsi donc pour cette recherche visant l'évaluation d'impact de la gestation et de non gestation sur certains paramètres hématologiques et biochimiques, nous nous sommes intéressés à l'hématocrite, le taux d'hémoglobine et les teneurs martiales dans le sang hémolysé, le sérum et les globules rouges. Ces potentialités hématologiques et martiales seraient-elles influencées par l'état physiologique de gestation et de non gestation?

Cette recherche a comme intérêt d'évaluer les possibilités d'anémie ferriprive et d'envisager une supplémentation conséquente en tenant compte de l'état physiologique surtout en zone tropicale. 


\section{MATERIEL ET METHODES}

Milieu : cette recherche a été réalisée sur les caprin des cliniques vétérinaires de Lubumbashi et ceux abattues au marché Mzee Kabila à Lubumbashi (zone tropicale) en République Démocratique du Congo. Les dosages de l'hématocrite et du taux d'hémoglobine ont été réalisé au laboratoire du service de physiologique tandis que le dosage martial était réalisé au laboratoire du service de biochimie de la faculté de Médecine Vétérinaire de l'Université de Lubumbashi (UNILU).

Animaux : Nous nous sommes servi du sang total et des sérums des 38 chèvres dont 18 gestantes et 20 non gestantes.

Matériel : Trente huit (38) échantillons du sang total prélevés sur EDTA, étaient utilisés pour la détermination de l'hématocrite, du taux d'hémoglobine et des teneurs martiales après hémolyse. Trente huit (38) autres échantillons sous forme du sérum étaient utilisés pour la détermination des teneurs martiales sériques. Pour le prélèvement, l'obtention de sérum, l'hémolyse et la conservation des échantillons, nous nous sommes sévi des seringues, couteaux, tubes à essais, EDTA comme anticoagulant, réfrigérateur et centrifugeuse. L'hémoglobinomètre de Sahli, ses annexes et $\operatorname{HCL}(0,1 \mathrm{~N})$ était utilisés pour le dosage du taux de l'hémoglobine tandis que les micropipettes et micro centrifugeuse, pour la détermination de l'hématocrite. Quant au dosage du fer, nous avons utilisé le spectrophotomètre et le kit du fer recommandé par la maison commerciale cypress diagnostic Belgique.

\section{RESULTAT :}

Les résultats de cette recherche sont résumés dans 4 tableaux ci-après :

Tableau 1: L'hématocrite (\%), le taux l'hémoglobine $(\mathrm{g} \%)$ et les teneurs martiales sériques, du sang totales et globulaires $(\mu \mathrm{g} / \mathrm{dl})$ des chèvres gestantes.

\begin{tabular}{|c|c|c|c|c|c|}
\hline No & Hématocrite & hémoglobine & Fer sérique & Fer total & Fer globulaire \\
\hline 1 & 30 & 5 & 221,96 & 304,10 & 81,14 \\
\hline 2 & 35 & 7 & 79,37 & 533,33 & 453,96 \\
\hline 3 & 21 & 3,8 & 65,89 & 450,31 & 384,42 \\
\hline 4 & 38 & 7,8 & 6,05 & 400,61 & 394,61 \\
\hline 5 & 30 & 4 & 18,49 & 514,90 & 496,41 \\
\hline 6 & 40 & 8 & 1,79 & 493,20 & 491,41 \\
\hline 7 & 34 & 4 & 4,04 & 167,70 & 163,66 \\
\hline 8 & 45 & 7,4 & 32,28 & 481,48 & 449,2 \\
\hline 9 & 35 & 5,6 & 267,63 & 267,08 & 0,55 \\
\hline 10 & 40 & 7 & 44,17 & 557,03 & 512,86 \\
\hline 11 & 28 & 4,6 & 63,16 & 507,45 & 443,23 \\
\hline 12 & 45 & 10 & 16,14 & 461,81 & 445,67 \\
\hline 13 & 35 & 5,8 & 73,41 & 290,68 & 217,27 \\
\hline 14 & 40 & 7,6 & 4,48 & 475,30 & 470,82 \\
\hline 15 & 30 & 5,8 & 78,83 & 234,67 & 1161,84 \\
\hline 16 & 40 & 6,4 & 5,38 & 523,45 & 518,07 \\
\hline 17 & 25 & 5,6 & 31,10 & 1008,69 & 974,58 \\
\hline $\begin{array}{l}18 \\
N \\
M \pm S \\
\text { Fer en } \mu \mathrm{mol} / \mathrm{l} \\
\%\end{array}$ & $\begin{array}{l}45 \\
18 \\
35,33 \pm 6,98\end{array}$ & $\begin{array}{l}6,8 \\
18 \\
6,23 \pm 1,64\end{array}$ & $\begin{array}{l}170,85 \\
18 \\
65,72 \pm 77,70 \\
11,76 \pm 13,90 \\
15\end{array}$ & $\begin{array}{l}421,60 \\
18 \\
449,58 \pm 180,98 \\
80,47 \pm 32,39 \\
100\end{array}$ & $\begin{array}{l}250,75 \\
18 \\
383,91 \pm 218,70 \\
68,71 \pm 39,14 \\
85\end{array}$ \\
\hline
\end{tabular}

Légende : $M=$ moyenne, $S=$ écart-type, $\mathrm{N}=$ nombre d'échantillon

L'examen de ce tableau montre que l'hématocrite et le taux de l'hémoglobine des chèvres gestantes est de $35,33 \pm 6,98$ $\%$ et de $6,23 \pm 1,64 \mathrm{~g} \%$. Par rapport au fer du sang total, le taux du fer sérique est de $15 \%$ et de $85 \%$ pour le fer globulaire. 

hémoglobine et les teneurs martiales chez la chèvre a Lubumbashi en zone tropicale

\section{Méthodes}

Choix des animaux: Pour les chèvres des Cliniques vétérinaires, la sélection des animaux était basée sur examen physique à la vie, à la palpation et au constat du Berger (chevrier) alors que pour les chèvres du marché, la sélection était basée sur la présence ou absence du fœus à l'abattage.

Prélèvement: pour les chèvres des cliniques vétérinaires, 38 échantillons de sang dont 10 avec anticoagulant, 10 sans anticoagulant pour les chèvres non gestantes et, 9 avec anticoagulant, 9 sans anticoagulant pour les chèvres gestantes, ont été prélevé par ponction à la veines jugulaires ; 38 échantillons du sang des chèvres du marché étaient prélevés après détroncation des veines jugulaire. Huit( 8 ) à $10 \mathrm{ml}$ de sang dont 4 à $5 \mathrm{ml}$ ont été repartis dans des tubes avec et sans anticoagulant. Deux (2) ml de sérum du sang sans EDTA étaient tirés après centrifugation. Du sang avec EDTA était utilisé pour la détermination de l'hématocrite et du taux de l'hémoglobine.

Dosage du fer : Alors que la teneur du fer globulaire était la différence entre le fer du sang hémolysé et le fer sérique, la teneur du sang total hémolysé par action mécanique et par congélation et décongélation et celle du sérum était obtenu par la méthode colorimétrique "ferrosine " recommandée par la maison Cypress diagnostic Belgique.

Évaluation statistique: Pour l'évaluation statistique, nous avions procédé aux calculs des moyennes, écarttype, variances et pourcentages selon les méthodes classiques. Pour la comparaison des moyennes, nous nous sommes servis du test de $T$ de student tandis que pour le coefficient de corrélation, nous avons recouru au logiciel Excel version 2010. Les différences ont été déclarées significatives ou non au seuil de $0,05 \%$. Le facteur de conversion du fer en $\mu \mathrm{mol} / \mathrm{l}$ étant 0,179

Tableau 2 : L'hématocrite (\%), le taux de l'hémoglobine ( $\mathrm{g} \%$ ) et les teneurs martiales sériques, du sang totales et globulaires $(\mu \mathrm{g} / \mathrm{dl})$ des chèvres non gestantes.

\begin{tabular}{|c|c|c|c|c|c|}
\hline$n^{0}$ & Hématocrite & Hémoglobine & Fer sérique & Fer total & Fer globulaire \\
\hline 1 & 28 & 6 & 141,04 & 401,86 & 260,89 \\
\hline 2 & 38 & 6,8 & 94,21 & 409,14 & 314,93 \\
\hline 3 & 35 & 6,2 & 222,54 & 1211,80 & 989,26 \\
\hline 4 & 40 & 7 & 28,90 & 845,71 & 816,81 \\
\hline 5 & 30 & 5 & 53,75 & 147,82 & 94,07 \\
\hline 6 & 42 & 6 & 124,27 & 793,71 & 669,44 \\
\hline 7 & 30 & 6 & 102,89 & 1381,36 & 1278,47 \\
\hline 8 & 38 & 6,8 & 26,01 & 814,28 & 788,27 \\
\hline 9 & 35 & 5,6 & 174,56 & 425,46 & 250,9 \\
\hline 10 & 45 & 7 & 130,05 & 513,73 & 383,66 \\
\hline 12 & 32 & 5 & 9,24 & 1208,69 & 1199,45 \\
\hline 13 & 35 & 6,2 & 11,56 & 472,57 & 461,01 \\
\hline 14 & 35 & 5,4 & 121,96 & 1127,32 & 1005,36 \\
\hline 15 & 36 & 6 & 147,97 & 588 & 440,03 \\
\hline 15 & 35 & 5,5 & 201,15 & 777,01 & 575,86 \\
\hline 16 & 42 & 6,4 & 151,44 & 525,14 & 373,7 \\
\hline 17 & 30 & 5,8 & 120,23 & 474,53 & 354,3 \\
\hline 18 & 40 & 6 & 2,89 & 410,28 & 407,39 \\
\hline 19 & 25 & 4,8 & 19,07 & 416,77 & 354,3 \\
\hline $\begin{array}{l}20 \\
\mathrm{~N} \\
\mathrm{M} \pm \mathrm{S} \\
\text { Fer en } \mu \mathrm{mol} / \mathrm{l} \\
\%\end{array}$ & $\begin{array}{l}40 \\
20 \\
35,55 \pm 5,23\end{array}$ & $\begin{array}{l}6 \\
20 \\
5,98 \pm 0,64\end{array}$ & $\begin{array}{l}177,45 \\
20 \\
103,10 \pm 69,03 \\
18,45 \pm 12,35 \\
15\end{array}$ & $\begin{array}{l}680,57 \\
20 \\
683,59 \pm 329,85 \\
122,36 \pm 59,04 \\
100\end{array}$ & $\begin{array}{l}503,12 \\
20 \\
578,23 \pm 330,77 \\
103,50 \pm 59,20 \\
85\end{array}$ \\
\hline
\end{tabular}

L'examen de ce tableau montre que l'hématocrite et le taux de l'hémoglobine des chèvres non gestantes est respectivement de $35,55 \pm 5,23 \%$ et de $5,98 \pm 0,64 \mathrm{~g} \%$. Par rapport au fer du sang total, le taux du fer sérique est de $15 \%$ et de $85 \%$ pour le fer globulaire. 

hémoglobine et les teneurs martiales chez la chèvre a Lubumbashi en zone tropicale

Tableau 3 : Moyenne générale d'hématocrite (\%), taux d'hémoglobine ( $\mathrm{g} \%)$, fer du sang hémolysé, fer sérique et fer globulaire $(\mu \mathrm{mol} / \mathrm{l})$

\begin{tabular}{l|l|l|l|l|l}
\hline Catégories & hématocrite & Hémoglobine & Fer total & Fer sérique & Fer globulaire \\
\hline Gestantes & $35,33 \pm 6,98$ & $6,23 \pm 1,64$ & $80,47 \pm 32,39$ & $11,76 \pm 13,90$ & $68,71 \pm 39,14$ \\
\hline Non gestantes & $35,55 \pm 5,23$ & $5,98 \pm 0,64$ & $122,36 \pm 59,04$ & $18,45 \pm 12,35$ & $103,50 \pm 59,20$ \\
& & & & & \\
\hline
\end{tabular}

Ce tableau des moyennes générales ne révèle pas des différences significatives pour l'hématocrite et l'hémoglobine mais une supériorité significative de toutes les teneurs martiales des non gestantes par rapport aux gestantes

Tableau 4 : Coefficient de corrélation entre les différents paramètres hématologiques et biochimiques étudiés.

\begin{tabular}{l|l|l|l|l|l|l}
\hline Catégorie & Hct/Hb & Hct/FerS & Hct/FerG & Hb/FeS & Hb/FerG & FerS/FerG \\
\hline Gestantes & 0,78 & $-0,12$ & $-0,22$ & $-0,24$ & $-0,10$ & 0,46 \\
\hline Non gestantes & 0,69 & 0,15 & $-0,01$ & 0,07 & 0,00 & 0,01 \\
\hline
\end{tabular}

Légende : $\mathrm{Hb}=$ hémoglobine, $\mathrm{HCT}=$ hématocrite, FerS= fer sérique, FerG= fer globulaire.

L'examen de ce tableau montre que selon les paramètres étudiés, le coefficient de corrélation est positif et élevé entre hématocrite et hémoglobine pour les deux catégories, positif et faible entre le fer sérique et fer globulaire chez les gestantes, faible négatif entre le fer sériquel hémoglobine puis entre hématocrite/fer

\section{DISCUSSION}

Au cours de cette discussion, il sera question de comparaison et d'interprétation de l'hématocrite, du taux de l'hémoglobine et des teneurs martiales obtenus au cours de notre étude dans le sang total, sang hémolysé, sérum et globules rouges des chèvres gestantes et non gestantes exploitées sur pâturage des cliniques vétérinaires de Lubumbashi et celles abattues dans la tuerie du marché Mze à Lubumbashi en RDC.

Hématocrite : Les résultats présentés dans les tableaux I, II et III montrent que pour l'hématocrite variant entre 21 à $45 \%$, la valeur moyenne est de $35,33 \pm 6,68 \%$ chez les chèvres gestantes contre 25 à $45 \%$ pour une moyenne de $35,55 \pm 5,23 \%$ chez les chèvres non gestantes. La comparaison statistique de ces deux moyennes d'hématocrite n'a pas révélée des différences significatives, L'état de gestation n'a pas influencé ce paramètre alors qu'en principe l'hémodilution de gestation pouvait baisser sensiblement ce paramètre. Alors que pour les chèvres gestantes et non gestantes les moyennes d'hématocrite sont des $35,33 \pm 6,68 \%$ et de $35,55 \pm 5,23 \%$, comparable à $37,2 \pm 13,14$ obtenu chez les chèvres BET par Ndoutamia et Ganda (2005). Ces moyennes locales sont néanmoins supérieures à $27,5 \pm 0,7 \%$ obtenu chez les chèvres Kirdimi et de loin inférieures à $59,4 \pm 6 \%$ obtenu sur les chèvres Sahéliennes par les mêmes auteurs au Tchad. La variation de l'hématocrite local de 21 à $45 \%$ est globulaire. Elle est par contre inexistante chez les gestantes entre le fer sérique/hématocrite, hémoglobine/fer globulaire. Pour le non gestantes il y a inexistence de corrélation entre fer sérique/hématocrite, fer sérique/hémoglobine, Hématocrite/fer globulaire, Fer sérique/fer globulaire.

comparable à celle de 22-38\% avancé par Siliart et Nguyen (2007) chez la même espèce Bien que notre valeur maximale de $45 \%$ parait supérieure de leur valeur maximale de $38 \%$. La différence entre l'hématocrite local et ceux observés aux Tchad, dépendraient du degré d'abreuvement et de sudation dans les différentes zones tropicales.

Hémoglobine : Les résultats présentés dans les tableaux I, II et III montrent que le taux d'hémoglobine varie entre 3,8 à $10 \mathrm{~g} \%$ pour une moyenne de $6,23 \pm 1,64 \mathrm{~g} \%$ chez les gestantes et entre 4,8 à $7 \mathrm{~g} \%$ pour une moyenne de $5,98 \pm 0,64 \mathrm{~g} \%$ chez les non gestantes. L'examen statistique de ces deux moyennes, n'a pas révélé des différences significatives. L'état de gestation n'a pas influencé le taux d'hémoglobine alors que physiologiquement, l'hémodilution de gestation pouvait baisser le taux d'hémoglobine. Par rapport aux valeurs exotiques, le taux d'hémoglobine du type anémique observé localement, sont inferieur aux taux de $9 \pm 1,1 \mathrm{~g} \%$ et $12 \pm 1,3 g \%$ obtenu chez les chèvres du Tchad par Ndoutamia et Ganda (2005) et également inférieur au taux de 10 à $11 \mathrm{~g} \%$ avancées par Siliart et Nguyen (2007).

Teneurs martiales : Alors que les gestantes ont présenté des teneurs de $80,47 \pm 32,39 \mu \mathrm{mol} / \mathrm{l}$ pour le sang hémolysé, de $11,76 \pm 13,90 \mu \mathrm{mol} / \mathrm{l}$ pour le fer sérique et de $68,71 \pm 39,14 \mu \mathrm{mol} / \mathrm{l}$ pour le fer globulaire, Les non 
gestantes par contre ont présentées des teneurs martiales du sang hémolysé, du sérum et des globules rouges respectivement de 122,36 $\pm 59,04 \mu \mathrm{mol} / \mathrm{l}$, $18,45 \pm 12,35 \mu \mathrm{mol} / \mathrm{l}$ et de 103,50 $\pm 59,20 \mu \mathrm{mol} / \mathrm{l}$. La comparaison statistique des teneurs martiales correspondantes a relevée une supériorité globale martiale Par rapport aux gestantes. Malgré la mobilisation du fer hépatique, l'infériorité des teneurs martiales globale des gestantes par rapport aux non gestantes serait principalement liée à un important transfert du fer maternel vers le fœtus pour assurer à ce dernier l'érythropoïèse, la myoglobinogèse et divers processus métaboliques dont le système enzymatique (Ngoie et al, 2016). Chez les non gestantes, à part le transfert ferritinique dans le foie et la moelle osseuse, les pertes martiales réelles dans les urines, le lait et les desquamations sont faibles (Levy et al, 2008). Le dosage biochimique des différents paramètres dans le sang hémolysé étant généralement interdit pour ne pas fausser le diagnostic, et rare pour les composants biochimiques globulaires à part l'hémoglobine, la comparaison de nos résultats n'a pas était possible vis-à-vis des valeurs exotiques. Toutefois, les valeurs martiales sériques minimales observées localement, ont été de loin inferieures aux valeurs de $12,53 \mu \mathrm{mol} / / \mathrm{l}$ observées chez les bovins par Gustav (1979), de 17,9 $\mu \mathrm{mol} / \mathrm{l}$ observées par Blood et Henderson (1976) chez les ovins, de 40,50 $\mu \mathrm{mol} / \mathrm{l}$ observées chez les équins par Rajakoski et al. (1972) et de 8,9 $\mu \mathrm{mol} / / \mathrm{l}$ observée chez l'homme par Dennis et al (2006). Alors que Ngoie et al. (2016) avaient trouvaient que chez les porcs, le fer était plus concentré dans les globules rouges que dans le sérum, le taux de

\section{CONCLUSION et APPLICATION DES RÉSULTATS :}

La détermination de l'hématocrite et du taux d'hémoglobine des chèvres gestantes et non gestantes a montré que la gestation et la non gestation n'ont pas eu d'impact sur l'hématocrite et le taux d'hémoglobine. Le taux d'hémoglobine est du type anémie ferriprive. Par contre, la gestation réduit sensiblement les teneurs martiales du sang total, sérum et globules rouges. Les teneurs martiales globales chez les non gestantes étant significativement supérieures par rapport aux gestantes. La corrélation hématocrite hémoglobine est généralement

\section{REMERCIEMENTS}

Les auteurs remercient les Cliniques Vétérinaires de Lubumbashi, les assistants du service de biochimie normale et pathologique de la faculté de Médecine
$15 \%$ du fer dans le sérum et de $85 \%$ dans les globules rouges aussi bien chez les gestantes que chez les non gestantes, confirme les observation de Claude (2002). Cette baisse de teneurs martiales sériques observée localement serait la conséquence d'un faible apport alimentaire en fer ou d'un excès de perdition à la suite du parasitisme (Levy et al, 2008). Nos résultats chez les caprin confirment que la teneur du fer sérique est significativement plus faible par rapport à la teneur du fer globulaire.

Corrélation : Les résultats présentés dans le tableau IV montre que la corrélation est moyennement positive entre l'hématocrite et l'hémoglobine des gestantes et des non gestantes. En effet, cette corrélation moyenne et positive entre hématocrite et hémoglobines témoigne que le taux d'hémoglobine est proportionnel à l'hématocrite (Kolb, 1975). La corrélation positive et faible entre fer sérique et fer globulaire des gestantes montre que le fer sérique chez les gestantes, serait aussi véhiculé vers la moelle osseuse pour stimuler l'érythropoïèse et l'hémoglobinogenèse des gestante. La négativité du fer sérique par rapport à l'hémoglobine, montre que le fer sérique en cas de non mobilisation des réserves, diminue quand l'hémoglobinogenèse augmente. Chez les non gestantes, à part la corrélation hématocrite/hémoglobine, les corrélations fer sérique/hématocrite, fer sérique/hémoglobine, fer globulaire/hémoglobine n'existent pas. En effet, ce non corrélation témoigne que le fer sérique est plus mobilisé pour des réserves hépatiques que pour les paramètres hématologiques. Cela pourrait être aussi l'expression d'un déficit martial ou d'anémie ferriprive.

moyenne aussi bien chez les gestantes que chez les non gestantes. Le coefficient de corrélation entre le fer et d'autres paramètres montre que la corrélation est inexistante, la teneur du fer étant soit du type anémique ou soit que le fer est plus orienté vers les réserves hépatiques que vers la moelle osseuse. Nous recommandons aux éleveurs de supplémenter les chèvres surtout pendant la gestation pour lutter contre l'anémie qui menace les gestantes.

Vétérinaire de l'Université de Lubumbashi, ainsi que les statisticiens et informaticiens pour leurs apports à la réalisation de ce travail. 


\section{REFERENCES}

Barone R., 2010 : Anatomie comparée des mammifères domestiques, splanchnologie 2, tome 4, Vigotfrères, Paris.

Blood D.C. et Henderson J.A., 1976 : Médecine Vétérinaire, Vigot-frères, Paris.

Claude J.B., 2002: Introduction à la nutrition des animaux domestiques. Ed. Médicale internationale, Paris.

Dennis L. ; Kasper M.D. ; Eugen B. ; Anthony S., Fauci M.D., Dan L.; Jameson J.L.; Stephen L.H., 2006 : Principe de Médecine interne. Médecinescience Flammarion, Paris.

Gustav R., 1979: examen clinique des bovins, les éditions du point vétérinaire, Alfort.

Kolb E., 1975 : Physiologie des animaux domestiques, Ed. Vigot-frères, Paris.

Levy J.P.; Varet B.; Lefrère F.; Clauvel J.P., 2008: hématologie et transfusion. Elsevier Masson, les Maulin-eaux Cedex.

Lunumbi O.J.B.H., 2008. Recherche de la formule barymetrique adaptable à la chèvre africaine « Capra hircus » de 0 à 12 mois, à Lubumbashi et ses environs (RDC). Thèse d'Agrégation en Médecine Vétérinaire UNILU, Lubumbashi.

Makumyaviri A.M. et Kabamba F.K., 2006: Diagnostic des helminthes gastro-intestinaux chez les bovins élevés dans la ferme interdiocésaine de Mubinza au Kasaï occidental. Ann. Fac Méd. vét., UNILU, 18 : 30-31

Marpeau L. ; Lansac J. ; Teurnier F. ; Nguyen F., 2010 : traité d'obstétrique. Ed. Elsevier Masson.

Ndoutamia G. et Nganda K., 2005 : Détermination des paramètres hématologiques et biochimiques des petits ruminants du Tchad. Revue Méd. Vét., 156, 4,202-206.

Ngoie K. ; Baraka K.K. ; Kasereka S.B. ; Kaluendi C.M. ; Kaputo M., 2016. Évaluation et comparaison des teneurs martiales dans le sang total hémolysé, sérum et globules rouges chez les truies gestantes et porcelets en élevage tropical .Int. Pure App.Biosci. 4, $3: 216-222$.

Rajakoski E. Mero M.; Maija V., 1972: Serum and total iron bending capacity of the serum in trotter's, $\mathbf{4}$, 4: 223-224.

Siliart B. et Nguyen F., 2007 : le memento biologie du vétérinaire. Les éditions de points vétérinaires cedex-France.

Suttle N.F., 2010: Mineral nutrition of liverstock $4^{\text {th }}$ Edition. CABI. Walling ford, VK. 